\title{
Nonlinear Schrödinger equation solitons on quantum droplets
}

\author{
A. S. Carstea* \\ Department of Theoretical Physics, National Institute of Physics and Nuclear Engineering, Bucharest-Măgurele 077125, Romania \\ A. Ludu (1) ${ }^{\dagger}$ \\ Department of Mathematics, Embry-Riddle Aeronautical University, Daytona Beach, Florida 32114, USA
}

(Received 3 April 2021; accepted 7 July 2021; published 16 July 2021)

\begin{abstract}
Irrotational flow of a spherical thin liquid layer surrounding a rigid core is described using the defocusing nonlinear Schrödinger equation. Accordingly, azimuthal moving nonlinear waves are modeled by periodic dark solitons expressed by elliptic functions. In the quantum regime the algebraic Bethe ansatz is used in order to capture the energy levels of such motions, which we expect to be relevant for the dynamics of the nuclear clusters in deformed heavy nuclei surface modeled by quantum liquid drops. In order to validate the model we match our theoretical energy spectra with experimental results on energy, angular momentum, and parity for $\alpha$-particle clustering nuclei.
\end{abstract}

DOI: 10.1103/PhysRevResearch.3.033054

\section{INTRODUCTION}

Solitons are stable localized wave packets that can propagate a long distance in dispersive media without changing their shapes. Following the discovery of solitons by Russell [1], a large number of similar particle-like nonlinear localized waves, pulses, and finite-gap potentials were identified and discovered, influencing the development of almost all traditional areas of science, and also shaping modern fields of research [2]. Solitons are studied in a wide range of scales from cosmology and dark matter $[3,4]$ to the quantum scale [5] and new states of matter [6-8], and they occur in a broad spectrum of systems, from low temperature [9] to nonlinear biological or social systems [10]. Soliton theory initiated major developments in optical communication [11], especially by revealing universality properties of several nonlinear phenomena like rogue waves [12,13], in anomalous materials [14], or in the collective dynamics of large random ensembles (soliton gas, soliton rain) $[15,16]$. Solitons helped the development of new applications in technology: soliton computing [17], machine learning [18], or non-Hermitian optics [19]. At present, the long-range soliton stability is so well understood that ordered sets of solitons are used to carry out the transmission of information in fiber optics communication links [20].

Equivalently, the question of long-lifetime solitons confined in a compact region $[21,22]$ represents a subject of active research in ocean wave dynamics. Solitons are present not

\footnotetext{
*acarst@ theory.nipne.ro

†ludua@erau.edu

Published by the American Physical Society under the terms of the Creative Commons Attribution 4.0 International license. Further distribution of this work must maintain attribution to the author(s) and the published article's title, journal citation, and DOI.
}

only in long and narrow geometries such as channels, fiber optics, electric lines, or nerves, but they were also found as soliton gas or periodic waves in compact regions [22] and in bounded nonlinear optics systems [12,15]. A rain of soliton pulses, triggered by a noisy background, can start flowing inside a finite fiber laser cavity, together with its condensed phase [16]. Such trains of bound solitons (soliton molecules [23]) can also travel at constant angular frequency through circular fiber rings [24]. It was also possible to generate multisoliton rotating clusters and quasipolygonal stable soliton clusters in bulk nonlinear optical media [25].

Rotating solitons/solitary waves can occur in microscopic systems. Such excitations are theoretically obtained in the quantum Hall effect of 2D electron drops [26] or in Bose-Einstein condensates [27], and they were measured in superfluid helium rotating vortices [28].

At laboratory scale, the formation of periodic nonlinear waves, or cnoidal waves for Korteweg-de Vries models $(\mathrm{KdV})$, on closed and bounded systems was detected, and the results were matched with theoretical calculations in lowtemperature interfacial systems [28,29], in confined rotating flows [30], and along circular chains of magnetic pendulums [31]. Experiments demonstrate the formation of rotating hollow polygons in 2D fluids, within a good match with theoretical models of cnoidal waves [29,32-39].

Cnoidal patterns and solitary waves at large scales were observed as vortex waves [40] and as rotating polygons in the hurricane eye wall [41], as well as in the case of Saturn's North Pole hexagon [42]. Numerical simulations for the azimuthal nonlinear surface waves on neutron stars surrounding a rigid core generate localized, shock-type dispersionless solutions [43].

The formation of solitons on spherical surfaces was considered as a possible explanation of large-amplitude collective modes of excitation on nuclear surfaces in the liquid drop model for cluster radioactivity [44] or as shape solitons on the 
surface of liquid drops [21]. Nonlinear models with soliton solutions offer possible explanations for the emergence of such rotons as coherent states in nuclear systems [45], in $\alpha$-particles' collision with medium-heavy nuclei [46-48], in nuclear fission [49], and in cnoidal excitations of Fermi-PastaUlam rings [50].

These results suggest that some dynamical systems can have collective localized stable excitations in compact or bounded geometries. Given the observed similarity between such rotating solitary waves within various ranges of physical scales (from nuclei to neutron stars) there may be a possibility of manifestation of signatures of universality.

In this paper we show that for a spherical thin liquid droplet surrounding a rigid core one can develop an asymptotic procedure which gives the evolution of periodic envelope solitons in the azimuthal direction (the spherical $\varphi$ coordinate). The variation in the polar coordinate $\theta$ is considered to be very slow (more precisely this approximation is valid not very close to the spherical poles). The asymptotic (related to the thickness of the spherical fluid layer) of Laplace equations and kinematic boundary condition transforms the linearized spherical Euler equation into a nonlinear one, supporting plane wave solutions with a Boussinesq-type dispersion relation. In the full nonlinear Euler equation we assume that in a stretched space-time scale, a slow modulation of the plane wave occurs, and accordingly, a defocusing nonlinear Schrödinger equation is obtained. Periodic dark solitons solutions expressed by elliptic functions are described. In a sense, this paper is a continuation of [21] where cnoidal $\mathrm{KdV} 1$-phase solutions were founded. In Sec. III the last part we analyze the quantum dynamics of such system using an algebraic Bethe ansatz, a well-known procedure for the defocusing nonlinear Schrödinger equation. We believe that this fact to be relevant in the study of collective excitations of the surface of heavy nuclei in exotic radioactivity processes [44]. In the last section we match our theoretical energy spectra with experimental results on energy, angular momentum, and parity for $\alpha$-particle clustering nuclei for atomic masses ranging from 20 to 212 .

\section{GENERAL DERIVATION OF THE NONLINEAR SCHRÖDINGER EQUATION}

Solitons represent fundamental nonlinear modes of physical systems described by a special class of wave equations of an integrable nature. These equations, like the $\mathrm{KdV}$ equation or the nonlinear Schrödinger equation (NLS), are of significant physical importance since they describe at the leading order the behavior of many systems in various fields of physics

Our model is an ideal spherical liquid layer exhibiting irrotational flow. The inner surface is bounded by a rigid core of radius $R_{0}-h$, and the variable outer surface $\Sigma$ is paramaterized by spherical coordinates $r=R_{0}[1+\xi(\theta, \phi, t)]$. We further assume that traveling perturbations will be slowly varying in $\theta$ and the fast dynamics is happening in the $\phi$ direction, and so we separate $\xi(\theta, \phi, t)=g(\theta) \eta(\phi, t)$, with $g(\theta)$ a slowly varying function. From the equation of continuity for incompressible fluid $\rho=$ const and irrotational condition we have the Laplace $\Delta \Phi=0$ and Euler equation:

$$
\left(\Phi_{t}+\frac{1}{2}|\nabla \Phi|^{2}\right)_{\Sigma}=-\frac{P}{\rho}
$$

where $P$ is the pressure and $\Phi$ is the velocity potential. The boundary condition on $\Sigma$,

$$
\left.\frac{d r}{d t}\right|_{\Sigma}=\left(\partial_{t} r+\frac{d \theta}{d t} \partial_{\theta} r+\frac{d \phi}{d t} \partial_{\phi} r\right)_{\Sigma},
$$

can be written in terms of the velocity potential in spherical coordinates:

$$
\left.\Phi_{r}\right|_{\Sigma}=R_{0}\left(\xi_{t}+\frac{\xi_{\theta}}{r^{2}} \Phi_{\theta}+\frac{\xi_{\phi}}{r^{2} \sin ^{2} \theta} \Phi_{\phi}\right)_{\Sigma} .
$$

Because our model is a liquid shell we have the inner boundary condition $v_{r}=\left.\partial_{r} \Phi\right|_{r=R_{0}-h}=0$. In order to meet the harmonic condition we expand the flow potential $[21,22]$

$$
\Phi=\sum_{n=0}^{\infty}\left(\frac{r-R_{0}}{R_{0}}\right)^{n} f_{n}(\theta, \phi, t)
$$

where the functions $f_{n}$ must obey recursion relations obtained form the Laplace equation. Assuming the smallness parameter $h / R_{0}=\epsilon<<1$ and $\left(r-R_{0}\right) / R_{0}=\epsilon$ we obtain the following relations in the dominant order from the inner boundary condition:

$$
f_{1}=2 \epsilon f_{2}, \quad f_{2}=-\frac{1}{2}\left(\Delta_{\Omega} f_{0}+2 f_{1}\right) .
$$

From the free surface boundary condition and slowly variation on $\theta$ we can write

$$
f_{0, \phi}=\frac{R_{0}^{2} \sin ^{2} \theta \xi \xi_{t}}{\epsilon \xi_{\phi}}+O\left(\xi^{2}\right) .
$$

Also using the expansion of velocity potential we have in the first order

$$
\begin{gathered}
\Phi=f_{0}+\xi f_{1}+O\left(\xi^{2}\right), \quad \Phi_{\phi}=f_{0, \phi}+O\left(\xi^{2}\right), \\
v_{\phi}=\frac{\Phi_{\phi}}{r \sin \theta}=\frac{f_{0, \phi}}{R_{0} \sin \theta} .
\end{gathered}
$$

Deriving with respect to $\phi$ the Euler equation we get (we neglect the $\theta$ derivatives)

$$
\partial_{t}\left(f_{0, \phi}+\xi f_{1, \phi}+\cdots\right)+\partial_{\phi}\left(\frac{v_{\phi}^{2}}{2}\right)=\frac{2 \sigma}{\rho R_{0}} \xi_{\phi}+\frac{\sigma}{\rho R_{0}} \Delta_{\Omega} \xi_{\phi}+O\left(\xi^{2}\right),
$$

we obtain

$$
\partial_{t}\left(\frac{R_{0}^{2} \sin ^{2} \theta \xi \xi_{t}}{\epsilon \xi_{\phi}}\right)+\partial_{\phi}\left(\frac{R_{0}^{2} \sin ^{2} \theta \xi^{2} \xi_{t}^{2}}{2 \epsilon^{2} \xi_{\phi}^{2}}\right)-\frac{2 \sigma \xi_{\phi}}{\rho R_{0}}-\frac{\sigma \xi_{\phi \phi \phi}}{\rho R_{0} \sin ^{2} \theta}=0
$$


The linearized version of the Euler equation is given by

$$
\Phi_{t}=-\frac{1}{\rho} P,
$$

which further can be written

$$
\partial_{t}\left(\frac{R_{0}^{2} \sin ^{2} \theta \xi \xi_{t}}{\epsilon \xi_{\phi}}\right)=\frac{2 \sigma}{\rho R_{0}} \xi_{\phi}+\frac{\sigma}{\rho R_{0} \sin ^{2} \theta} \xi_{\phi \phi \phi} .
$$

This equation admits the linear traveling wave solution $\xi=A(\theta) e^{i(k \phi-\omega t)}+$ c.c. with the Boussinesq-type dispersion relation

$$
\omega^{2}=\frac{\epsilon \sigma}{\rho R_{0}^{3} \sin ^{2} \theta}\left(2 k^{2}-\frac{k^{4}}{\sin ^{2} \theta}\right) .
$$

In the long-wave limit $k=\epsilon K \sim O(\epsilon)$ the dispersion relation becomes

$$
\begin{gathered}
\omega(K)=\frac{\epsilon^{1 / 2}}{R_{0} \sin \theta} \sqrt{\frac{2 \sigma}{\rho R_{0}}}\left(\epsilon K-\frac{\epsilon^{3} K^{3}}{4 \sin ^{2} \theta}\right)+O\left(K^{5}\right) \\
\equiv \epsilon v K+\beta \epsilon^{7 / 2} K^{3}+O\left(K^{11 / 2}\right),
\end{gathered}
$$

with $v=\epsilon^{1 / 2} /\left(R_{0} \sin \theta\right) \sqrt{2 \sigma / \rho R_{0}}$ being the phase velocity. It results that for the monochromatic case this dispersion provides exactly the stretched variables for the $\mathrm{KdV}$ equation. Indeed, from Eq. (3) we have

$$
\begin{aligned}
\xi= & A \exp [i(k x-\omega t)]=A \exp \{i K[\epsilon(\phi-v t) \\
& \left.\left.-\epsilon^{7 / 2} \beta K^{3} t-\cdots\right]\right\},
\end{aligned}
$$

and this suggests the variables $\phi \rightarrow \epsilon(\phi-v t), T \sim$ $\epsilon^{7 / 2} t, \xi \rightarrow \epsilon^{3} g(\theta) \eta(\phi, T)$. In these new variables one obtains immediately the $\mathrm{KdV}$ equation in $\eta(\phi, T)$, which is analyzed extensively in $[21,22]$ In the following, one can see that for $\xi(\phi, t)=A e^{i(k \phi-\omega t)}$ we have $\xi \xi_{t} / \xi_{\phi}=-(\omega / k) \xi \equiv-v \xi$. This nonlinearity produces higher harmonics and weak modulation of amplitude in the slow variables $\varphi, \tau$, which will be defined next. So we make the following approximation:

$$
\frac{\xi \xi_{t}}{\xi_{\phi}} \sim \xi(\phi, \theta, t)=\sum_{n=-\infty}^{\infty} \varepsilon^{s_{n}} Q_{n}(\varphi, \tau, \theta) e^{i n(k \phi-\omega t)},
$$

where $\varepsilon$ is a small parameter measuring the weak modulation of the amplitude in a slow space-time scale (different form $\left.\epsilon=h / R_{0}\right)$ and $s_{n}$ are some exponents which have to be determined form balance. Now we can define the slow variables

$$
\begin{aligned}
\varphi & =\varepsilon\left(\phi-\frac{2 \sigma t}{v \rho R_{0}^{2} \sin ^{2} \theta}+\frac{3 \sigma k^{2} t}{v \rho R_{0}^{3} \sin ^{4} \theta}\right), \\
\tau & =-\varepsilon^{2} k t,
\end{aligned}
$$

and the amplitudes of the expansion:

$$
\begin{aligned}
& Q_{0}(\varphi, \theta, \tau)=\varepsilon^{2} g(\theta) V_{0}(\varphi, \tau), \\
& Q_{2}(\varphi, \theta, \tau)=\varepsilon^{2} g(\theta) V_{2}(\varphi, \tau), \\
& Q_{n}(\varphi, \theta, \tau)=\varepsilon^{n} g(\theta) V_{n}(\varphi, \tau), \quad n \neq 0,2, \quad V_{-n}=V_{n}^{*} .
\end{aligned}
$$

Introducing these expressions from above in the Euler equation we obtain the following defocusing nonlinear
Schrödinger (NLS) equation with dimensionless terms:

$$
i \frac{A(\theta)}{3 D(\theta)} \frac{\partial \zeta}{\partial \tau}+\frac{\partial^{2} \zeta}{\partial \varphi^{2}}-\frac{C(\theta)^{2} g(\theta)^{2}}{18 D(\theta)^{2}}|\zeta|^{2} \zeta=0
$$

where we used the following notations:

$$
\begin{aligned}
\zeta(\varphi, \tau) & =\frac{V_{1}}{k}, A(\theta)=\frac{v R_{0}^{2} \sin ^{2} \theta}{h}, \\
C(\theta) & =\frac{v^{2} R_{0}^{4} \sin ^{4} \theta}{h^{2}}, D(\theta)=-\frac{\sigma}{\rho R_{0} \sin ^{2} \theta} .
\end{aligned}
$$

The physical configuration is give by the parametrization equation

$$
\begin{aligned}
r= & R_{0}\left\{1+g(\theta)\left[\epsilon k \zeta(\varphi, \tau) e^{i(k \phi+\omega t)}+\right.\text { c.c. }\right. \\
& \left.\left.+\epsilon^{2}\left(\frac{C g}{6 D} \zeta^{2}-\frac{C g}{3 D}|\zeta|^{2} e^{2 i(k \phi+\omega t)}+\text { c.c. }\right)\right]\right\}
\end{aligned}
$$

\section{Dark periodic soliton}

In order to obtain periodic solutions and traveling waves, we consider

$$
\zeta(\varphi, \tau)=f(k \varphi+\omega \tau) e^{i(\lambda \varphi+\Omega \tau)},
$$

and we make the shorthand notations $s=k \varphi+\omega \tau$ and $\eta=$ $\lambda \varphi+\Omega \tau$. By introducing these notations in the NLS equation (4) it results in

$$
\begin{gathered}
\left(18 D \lambda^{2}+6 A \Omega\right) f(s)-6 i(6 D k \lambda+A \omega) f^{\prime}(s) \\
+C^{2} D g^{2} f(s)^{3}-18 D k^{2} f^{\prime \prime}(s)=0 .
\end{gathered}
$$

By imposing $6 D k \lambda+A \omega=0$ we obtain an equation which can be solved by elliptic functions. To make it simpler we divide by $18 D k^{2}$, and we find

$$
b_{0} f(s)+b_{1} f(s)^{3}-f^{\prime \prime}(s)=0,
$$

where

$$
b_{0}=\left(\frac{\lambda}{k}\right)^{2}+\frac{A \Omega}{3 D k^{2}}, \quad b_{1}=-\frac{C^{2} g^{2}}{18 k^{2}}, \quad \omega=-\frac{6 D k \lambda}{A} .
$$

The solution of Eq. (6) is

$$
f(s) \equiv f(\varphi, \tau)=H \operatorname{sn}\left[k \sqrt{\frac{C^{2} g^{2}}{18 k^{2}(m+1)}}\left(\varphi-\frac{6 D \lambda}{A} \tau\right) \mid m\right],
$$

where $H=i \sqrt{2 b_{1} m / b_{0}(m+1)}$. When $m \rightarrow 1$ we obtain the dark line-soliton limit

$$
f(\varphi, \tau) \rightarrow i \sqrt{\frac{b_{1}}{b_{0}}} \tanh \left[\sqrt{\frac{-b_{1}}{2}} k\left(\varphi-\frac{6 D \lambda}{A} \tau\right)\right] .
$$

We stress that the solution in Eq. (7) is a particular one. The most general solution has the form

$$
\zeta(\varphi-v \tau)=\sqrt{f(\varphi-v \tau)} \exp [\operatorname{ig}(\varphi-v \tau)]
$$


and it can be expressed in terms of the Jacobi sn function and the elliptic integral of the third kind

$$
\begin{aligned}
f(x)= & a_{1}+\left(a_{2}-a_{1}\right) \operatorname{sn}^{2}\left[\sqrt{\frac{c\left(a_{3}-a_{1}\right)}{2}} x \mid m\right], \\
g(x)= & \frac{\nu x}{2}+\sqrt{\frac{a_{2} a_{3}}{a_{1} a_{3}-a_{1}^{2}}} \Pi \\
& \times\left\{1-\frac{a_{2}}{a_{1}} ; \operatorname{am}\left[\sqrt{\frac{c\left(a_{3}-a_{1}\right)}{2}} x\right] \mid m\right\},
\end{aligned}
$$

where $a_{1}, a_{2}, a_{3}$ are the roots of the "potential" equation related to $f$ and $m=\sqrt{\left(a_{2}-a_{1}\right) /\left(a_{3}-a_{1}\right)}$; see [51] for details.

\section{QUANTIZATION}

Our equation (4) can be written in a Hamiltonian form:

$$
\partial_{T} \zeta=\frac{\delta}{\delta \zeta^{\dagger}} \int_{0}^{2 \pi}\left(\left|\zeta_{\varphi}\right|^{2}+c|\zeta|^{4}\right) d \varphi
$$

where we rescaled time $T=(A / 3 D) \tau$, and $c=C^{2} g^{2} / 36 D^{2}$. The pseudovacuum is $|0\rangle$ and $\zeta(\varphi)|0\rangle=0$. In order to perform the quantization, we discretize the system on a lattice, which means that our system will not be defined anymore on the meridian circle of the spheroidal drop, but on a polygon with $M$ sides. Also the evolution variable is the angle $\varphi:=n$, which is increased or decreased by fixed step angle $h$. A Lax operator with $\lambda$ spectral parameter is [52]

$$
L_{n}(\lambda)=\left(\begin{array}{cc}
1-\frac{i \lambda h}{2} & -i h \sqrt{c} \zeta_{n}^{\dagger} \\
i h \sqrt{c} \zeta_{n} & 1+\frac{i \lambda h}{2}
\end{array}\right)+O\left(h^{2}\right),
$$

and the quantum operators obey $\left[\zeta_{n}, \zeta_{m}^{\dagger}\right]=\delta_{n m} / h$, where we consider that $\hbar \equiv 1$.

Because we have periodic boundary condition the Lax operator is transformed to a monodromy operator. Namely, by imposing periodicity we have the transition from a zerocurvature formulation to the pure Lax formulation by using the monodromy matrix

$$
T(\lambda)=L_{M}(\lambda) \cdots L_{1}(\lambda)=\left(\begin{array}{ll}
A(\lambda) & B(\lambda) \\
C(\lambda) & D(\lambda)
\end{array}\right),
$$

where $A, B, C, D$ are operators, and not the coefficients of the initial KdV or NLS equations. The evolution of the Lax operator can be written either with a new matrix $P$ in the form $L_{t}=[P, L]$ or equivalently using the R-matrix formalism, which singles out the Hamiltonian structure. When we quantize, we can write explicitly the commutation relation between elements of monodromy matrix using the so-called RTT relation

$$
R(\lambda, \mu)[T(\lambda) \otimes T(\mu)]=[T(\mu) \otimes T(\lambda)] R(\lambda, \mu),
$$

where the matrix $R$ is given by

$$
R=\left(\begin{array}{cccc}
f(\lambda, \mu) & 0 & 0 & 0 \\
0 & g(\lambda, \mu) & 1 & 0 \\
0 & 1 & g(\lambda, \mu) & 0 \\
0 & 0 & 0 & f(\lambda, \mu)
\end{array}\right),
$$

with $f(\lambda, \mu)=1+i c /(\mu-\lambda), g(\lambda, \mu)=i c /(\mu-\lambda)$. Here $2 c$ is the $\theta$-dependent coefficient of our NLS equation (4), $C^{2} g^{2} / 18 D^{2}$. The action of elements of the monodromy matrix on the vacuum is

$$
\begin{aligned}
& A(\lambda)|0\rangle=a(\lambda)|0\rangle, D(\lambda)|0\rangle=d(\lambda)|0\rangle, \\
& C(\lambda)|0\rangle=0, B(\lambda)|0\rangle=\text { free. }
\end{aligned}
$$

As a result, one can see that in our case

$$
\begin{aligned}
a(\lambda) & =\prod^{M}(1-i \lambda h / 2)=(1-i \lambda h / 2)^{M}, \\
\lim _{M \rightarrow \infty} a(\lambda) & =e^{-i \lambda M h / 2}, d(\lambda)=(1+i \lambda h / 2)^{M}, \\
\lim _{M \rightarrow \infty}(1+i \lambda h / 2)^{M} & =e^{i \lambda M h / 2} .
\end{aligned}
$$

We can further use $M h \rightarrow 2 \pi$, since the full periodicity is of $2 \pi$ angle. The quantum states are constructed by applying the operator $B\left(\lambda_{i}\right)$ from the monodromy matrix. In the case of $N$ parameters (usually called rapidities) we have

$$
\Psi\left(\lambda_{1}, \ldots, \lambda_{N}\right)=\prod_{j=1}^{N} B\left(\lambda_{j}\right)|0\rangle .
$$

Now imposing that this $\Psi$ must be an eigenvector of the trace of the monodromy matrix we find the following Bethe equation:

$$
e^{2 \pi i \lambda_{m}}=\prod_{j=1, j \neq m}^{N}\left(\frac{\lambda_{m}-\lambda_{j}+i \frac{C^{2} g^{2}}{36 D^{2}}}{\lambda_{m}-\lambda_{j}-i \frac{C^{2} g^{2}}{36 D^{2}}}\right),
$$

and the eigenvalue of $\operatorname{Trace} T(\mu)$ are

$$
\operatorname{Trace} T(\mu) \Psi=[A(\mu)+D(\mu)] \Psi=\Lambda \Psi,
$$

with

$$
\Lambda=e^{-i \mu \pi} \prod_{j=1}^{N} f\left(\mu, \lambda_{j}\right)+e^{i \mu \pi} \prod_{j=1}^{N} f\left(\lambda_{j}, \mu\right),
$$

where, as was shown above, $f(\mu, \lambda)=1+i c /(\lambda-\mu)$. It is easy to note that all the quasi-momenta $\lambda_{j}$ are dimensionless. Since it is well known that the trace of the monodromy matrix is nothing but the generating function of conserved integrals of motion (as a power series in $1 / \mu$ in our case), we can finally write the eigenvalues of the Hamiltonian

$$
E_{N}=\sum_{m=1}^{N} \lambda_{m}^{2}
$$

where $N$ is the number of particles associated with $\Psi$, and $\lambda_{m}$ are the solutions of the transcendental Bethe equations (9), so we will have quantum levels parameterized by the polar angle $c=c(\theta)$ with the real values

$$
E_{N}(c)=\frac{\hbar^{2}}{R_{0}^{2}} \sum_{j=1}^{N} \lambda_{j}^{2}(c) .
$$

Equation (9) has only real solutions, and they are all periodic of period 1, so it is enough to consider the solutions $\lambda_{j} \in[0,1]$. 


\section{DISCUSSION}

One can ask what is the role of dark solitons and how their dynamics is seen in the quantum regime. First, as we have seen, we obtained a nonlinear Schrödinger equation with defocusing nonlinearity. On the spatial infinite line the soliton solutions are rarefaction (dark) nonlinear waves which are build upon a finite condensate. But for the periodic boundary conditions these rarefaction waves are turned into periodic solitons expressed through Jacobi elliptic functions. They describe periodic envelopes of azimuthal excitations with various periodicities. In the quantum regime the defocusing nonlinear Schrödinger equation is nothing but interacting delta-Bose gas (with periodic boundary conditions). The algebraic Bethe ansatz provides a quantization of the whole dynamical system described by the Hamiltonian (8) and not a quantization of a special classical solution (periodic dark soliton). However, there is a correspondence between the periodic dark soliton and the expectation value of the density operator on a special Bethe quantum state $\left\langle\Psi_{0}\left|\zeta^{\dagger}(\varphi, t) \zeta(\varphi, t)\right| \Psi_{0}\right\rangle[53]$ constructed using some specific Bethe numbers. The construction is complicated and involves numerical simulations. The structure of the energy spectra changes with $N$ and with the parameter $c=C^{2} g^{2} / 36 D^{2}$. For any given $N$ it is observed that there is always a region for the parameter $c=c_{\text {crit }}$ around which the energy spectrum becomes very dense. For values $c<c_{\text {crit }}$ the spectral lines are rather equidistant, while for larger $c>c_{\text {crit }}$ the spectrum tends to be quadratic, similar to the spectrum for the rigid rotor. The larger the number of eigenvalues $N$, the smaller the value of $c_{c r t}$. is. From the expression of the coefficients $C, D$ from liquid drop model introduced in [21] it results that $c \simeq 0$ at $\theta=0, \pi$ as expected since there are no soliton excitations orbiting at the poles of the droplet. In general, $c$ has its larger values around the equator $\theta \simeq \pi / 2$. For certain combinations between the soliton orbital speed $V$, the depth of the shallow layer $h$, and the strength of the surface tension coefficient $\sigma$, the parameter $c(\theta)$ can be very small for all polar angles $\theta$. For example, for fast solitons orbiting a very shallow layer $h / R_{0} \ll 1$ and for weak surface tension, one can have very small values of $c$, resulting in weak energy excitation, resulting in a small probability to excite such soliton solutions. For droplets of the size of a medium-heavy nucleus, the parameter $c$ acquires values around $c \sim 10^{3}$ for almost all values of $\theta$, resulting in a larger probability to excite such soliton excitations. Equation (9) can be rewritten in the form

$$
2 \pi \hat{\lambda}_{j}=2 c \sum_{j=1, j \neq N}^{N} \frac{\lambda_{j}-\lambda_{k}}{\left(\lambda_{j}-\lambda_{k}\right)^{2}-c^{2}},
$$

where the hat symbol represents the equivalence class modulo addition of integers $n_{j} \in \mathbb{Z}$. We can evaluate the solutions of this equation for large values of the parameter $c \gg \lambda$. In this case we can make the approximation $\left|\lambda_{j}-\lambda_{k}\right| \ll c$, and Eq. (11) becomes a linear system of equations in $\lambda_{j}$ with the free term given by a column of $N$ arbitrary integers $\left(n_{1}, \ldots, n_{j}, \ldots, n_{N}\right)^{T}$. It is straightforward to calculate the solutions of this linear system

$$
\lambda_{j} \simeq \frac{\sum_{k=1, k \neq j}^{N} n_{k}+(-1-\pi c-2 N) n_{j}}{\pi c(\pi c+N)} \simeq \frac{\hat{1}}{\pi c},
$$

and consequently, in this approximation, the spectrum has a quadratic structure

$$
E_{N} \simeq \sum_{k=1}^{N} \frac{n_{k}^{2}}{\pi^{2} c^{2}}, n_{k} \in \mathbb{Z}
$$

which is manifested for intermediate values for $c>\lambda$. Nevertheless, since $\lambda \in[0,1]$ there are limitations on the values of the arbitrary integers $n_{k}$ and in fact this constraint requires $n_{k}$ to be of order of the integer part of $(N-1) / 2$, meaning that all $n_{k}$ are constant and the spectrum is actually represented by a constant multiplied by a sum of ones. This observation explains the asymptotic behavior of the spectrum for large $c$ towards a harmonic oscillator spectrum. In fact, in the limit $c \rightarrow \infty$ Eq. (9) reduces to $\exp \left(2 \pi i \lambda_{j}\right)= \pm 1$ which generates an equidistant energy line spectrum, since $\hat{\lambda}_{j}=1 / 2$. The spectral density of the $\lambda_{j}$ solutions as a function of $c, N$ can be estimated by introducing a vector nonlinear operator $\mathfrak{O}=\left(\mathfrak{O}_{i}\right)$, acting on the $N$-dimensional unit cube of vectors $\vec{\lambda}=\left(\lambda_{1}, \ldots, \lambda_{N}\right)$ in the form

$$
\mathfrak{O}_{i}(\vec{\lambda})=\sum_{k=1, k \neq i}^{N} \frac{\lambda_{i}-\lambda_{k}}{\left(\lambda_{i}-\lambda_{k}\right)^{2}-c^{2}} .
$$

With this operator the Bethe ansatz Eq. (9) becomes a fixed point equation for this operator, and while acting on the vectors $\vec{\lambda}, \mathfrak{O}$ is a contraction, so it has a unique fixed point, and thus the eigenvector space reduces to one vector. For this case the energy spectrum has one spectral line only. Consequently, dense energy spectra are in the regions where this operator is not a contraction. For such regions inside the unit cube and for the corresponding values of $c$, the spectrum becomes rich in spectral lines. Obviously, when $|c|<1$ there are values for $\vec{\lambda}$ where the denominators in Eq. (11) approach zero, so the operator is described by a Lipschitz discontinued function, and thus $\mathfrak{O}$ cannot be a contraction. For these regions the spectrum becomes denser, as one can easily verify by numerical calculations in the region $c \simeq 1$. However we have to underline that in the limit of $c \rightarrow 0$ we have free bosons, while in the limit $c \rightarrow \infty$ the defocusing intercation is so huge and we have free fermions (inasmuch as the Bethe state obeys the Pauli principle). The Pauli principle for Bethe states shows that the nonlinear excitations of the quantum liquid drop model are purely fermionic.

\section{COMPARISON WITH NUCLEAR EXPERIMENTAL DATA}

In order to validate the physical relevance of the quantum nonlinear liquid drop representation introduced here, we compare the energy levels predicted by our model with experimentally measured resonant lines for $\alpha$-clustering nuclei [46-49,54-60]. Numerous studies show that $\alpha$ clustering occurs from light and medium-mass elements to heavy and superheavy elements. Various phenomenological and microscopic models have been proposed in the literature to describe various aspects of $\alpha$ clustering [54-60]. Among them, the large-amplitude nonlinear collective model [21,22,44-49,61] is of special interest for the present work. 


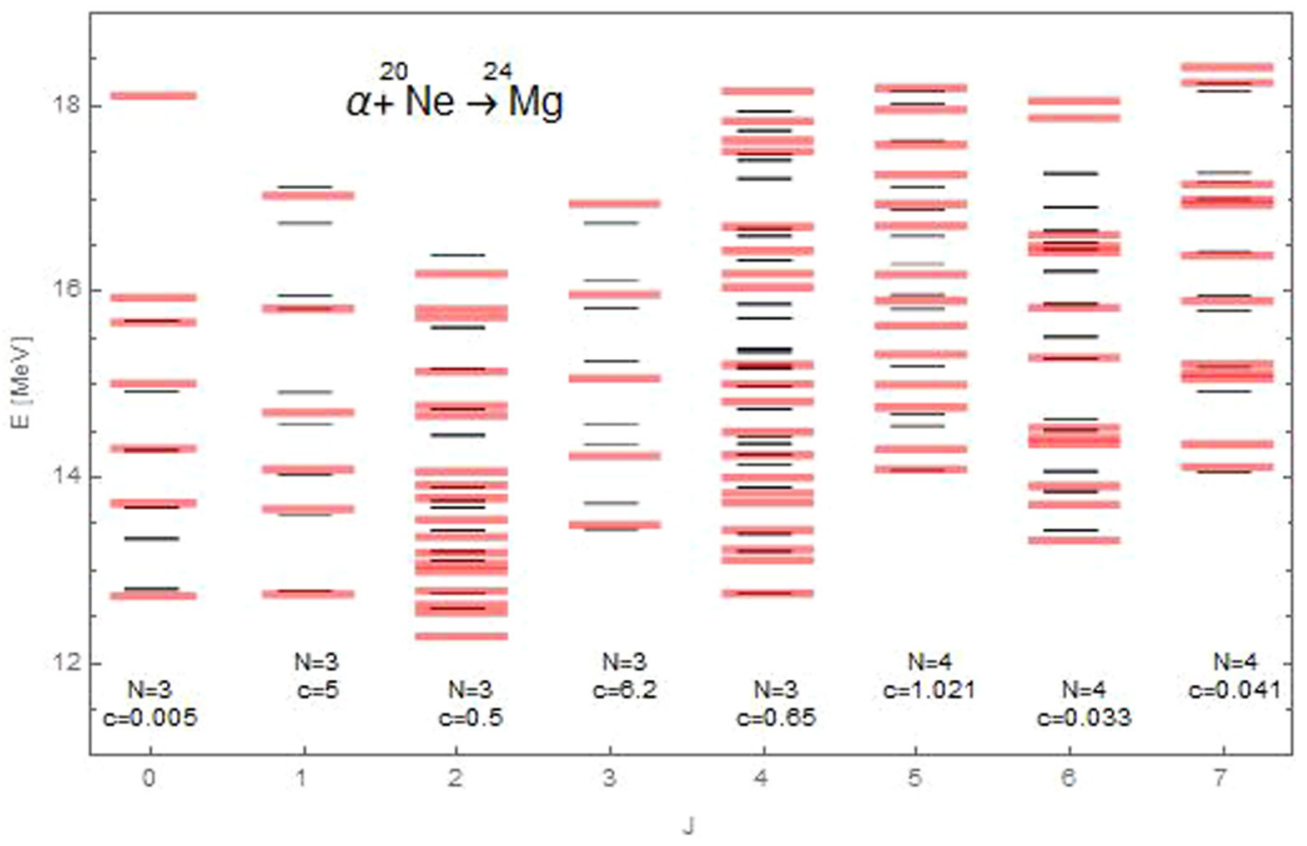

FIG. 1. Black: Experimental energy spectra, [47], of positive- and negative-parity resonant states obtained in the collision of $\alpha$-particles on ${ }^{20} \mathrm{Ne}$ targets with formation of bound $\alpha$-cluster states in ${ }^{24} \mathrm{Mg}$. The spectra are horizontally aligned by angular momentum $J$ from $J=00^{+}$ to $J=7^{-}$. Red: The theoretical Bethe spectra (10) are plotted for rapidities $N=3$ and 4 with the parameter $c$ chosen to provide the best fit with experiments. The odd angular momentum states (labeled with higher placed text in the figure) provide a good fit for larger values of $c$, typically $c>c_{\text {crit }}$, while the best fit for even states occur for relative smaller $c$, shown under each column.

In the following, we mention four classes of experimental observations and the associated theoretical questions, pointing our interest towards using nonlinear collective models. These types of models can relate the features of superdeformed nuclei, cluster radioactivity, quasimolecular structures, or $\alpha$ clustering to particular solutions of nonlinear evolution equations like Bose-Einstein condensation or solitons.

First, the experimental evidence of cluster decay as spontaneous emission of carbon, neon, magnesium, and silicon from heavy nuclei indicates a large enhancement of such clusters on the nuclear surface. By considering nonlinear terms in the hydrodynamics of the liquid drop model for the nucleus, it was inferred that KdV solitary waves could exist on the surface of nuclei and explain cluster decay as a large-amplitude collective excitation $[44,49,61]$. Nevertheless, in order to reproduce the experimental spectroscopic factors for $\alpha$ and cluster decays with such a nonlinear integrable model defined on the nuclear surface it was necessary to add shell corrections. Thus one obtained a coexistence model consisting of the usual shell model and a cluster-like model, leading to a minimum in the total potential energy degenerated with the ground state minimum.

Second, $\alpha$-like states were detected for many light to heavy nuclei. The $\alpha$ clustering in the nuclear structures and clustering models have a long history, but in the last decade a rapid development successfully explained the structure of many states in light to heavy nuclei, especially in $n \cdot \alpha$ nuclei [59].

Third, a moment of inertia anomaly was emphasized in the rotational bands of resonance elastic scattering measurements. By plotting the mean weighted values with the reduced widths of the experimental energy levels versus $J(J+1)$ for such experiments one can obtain a value for the moment of inertia of the system. By comparing this value with the theoretic moment of inertia of an $\alpha$-particle plus the daughter nucleus rotating together at a touching distance we have a discrepancy: The experiment provides smaller values by a factor of at least 2 than the rigid rotor moment of inertia [46,47,54]. For example, in [54] the measured moment of inertia for the elastic scattering $\alpha+{ }^{36} \mathrm{Ar} \rightarrow{ }^{40} \mathrm{Ca}$ was $\mathcal{I}=3.8 \pm 0.3 \hbar^{2} / \mathrm{MeV}$, while $\alpha$-particles orbiting a noninteracting ${ }^{36} \mathrm{Ar}$ core would have a $\mathcal{I}=9.3 \hbar^{2} / \mathrm{MeV}$ moment of inertia. Moreover, this larger theoretical value results also from calculations of the strongest superdeformed bands in ${ }^{40} \mathrm{Ca}(4 p-4 h$ and $8 p-8 h$ excitations). It appears that the geometric configuration of a cluster orbiting around a daughter nucleus is a little more complicated than a rigid rotor.

The fourth observation is related to various aspects of collective motion in nuclei. One of the collective motion degrees of freedom is caused by the spontaneous symmetry breaking of rotational invariance due to the $\alpha$ clustering. The collective motion related to cluster condensation, or superfluidity in nuclei, has received attention in the frame of the many-body theory in the last decades. In [55] it was shown that collective states of the zero mode operators are new-type soft modes due to Bose-Einstein condensate of $\alpha$ clusters.

From the Bose-Einstein phenomenological Hamiltonian of a number of $\alpha$-particles trapped by an external potential results a Gross-Pitaevskii equation (G-P) for the nuclear condensate component of the field operator. In the field theoretical superfluid cluster model, the solution of the G-P equation represents the order parameter of the phase transition from the Wigner phase to the Nambu-Goldstone phase. Because the order parameter is a superfluid amplitude, the square of the modulus of this amplitude represents the 
superfluid density distribution [55], which ultimately describes the nuclear shape. The G-P equation is in the same hierarchy as the NLS equation, just having in addition the potential trap linear term. Consequently, it is natural for the G-P equation to have solitary waves solutions as shown, for example, in $[2,7,8]$.

This final observation supports the NLS droplet model, since both G-P and NLS equations have a similar type of solitons and can be equally used to explain some exotic nuclear shapes. The NLS model for quantum droplets presented here has the advantage of being already quantized, so it does not request shell corrections when applied in nuclear models.

In all experimental comparison we use only three fitting parameters: $c$ (the shape parameter), $N$, and $E_{0}$, the last one being a multiplicative rescaling of the energy spectra in Eq. (10). The expression of the total energy is the sum between the scaled Bethe spectrum and a rigid rotation $E_{\text {exp }}=$ $E_{0} E_{N}(c)+\hbar^{2} J(J+1) /(2 \mathcal{I})$.

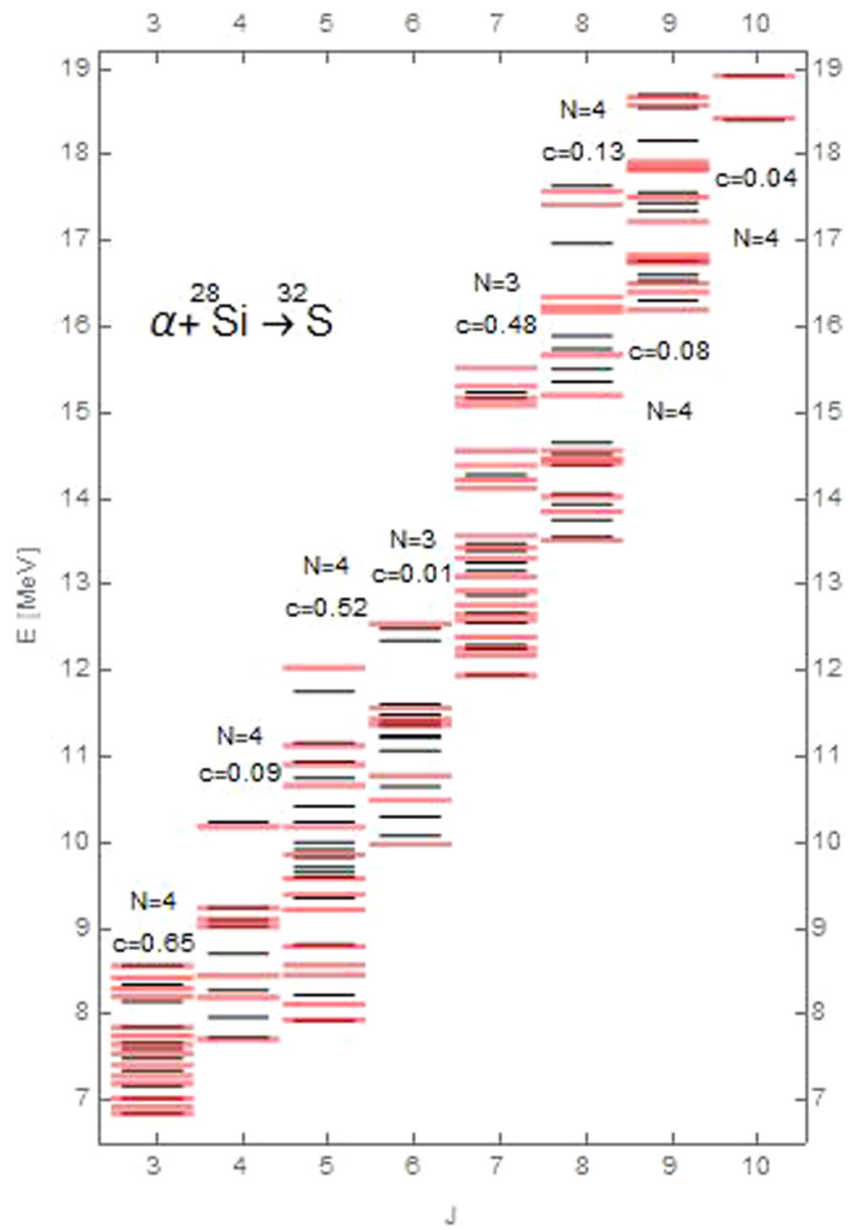

FIG. 2. Black: Experimental energy spectra, [46], of positiveand negative-parity resonant states obtained in the collision of $\alpha$-particles on ${ }^{28} \mathrm{Si}$ targets with formation of bound $\alpha$-cluster states in ${ }^{32} \mathrm{~S}$, plotted vs $J$ angular momentum. Red: The theoretical Bethe spectra (10) are plotted for $N=3$ and 4 with the parameter $c$ chosen to provide the best fit with experiments. The odd angular momentum states are again associated with larger values of $c$, shown in the figure on top of each column.

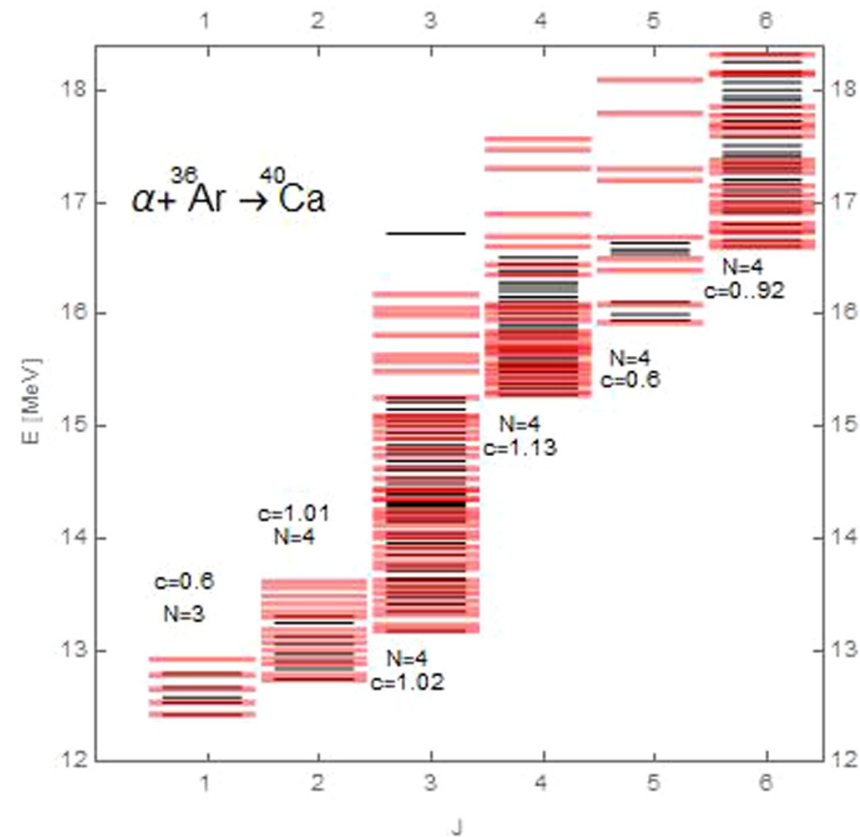

FIG. 3. Black: Experimental energy spectra, [54], of positiveand negative-parity resonant states, plotted vs $J$, measured during collisions of $\alpha$-particles on ${ }^{36} \mathrm{Ar}$ targets, with formation of bound $\alpha$-cluster states in ${ }^{40} \mathrm{Ca}$. Red: The theoretical Bethe spectra Eq. (10) for $N=3$ and 4 and $c$ values shown in the figure above or below each column.

In Fig. 1 we present a comparison between experimental spectra [47] of resonant states obtained in the collision of $\alpha+{ }^{20} \mathrm{Ne} \rightarrow{ }^{24} \mathrm{Mg}$ generating bound $\alpha$-cluster states $J=0^{+}$ to $J=7^{-}$and the theoretical Bethe spectra Eq. (10) for $N=3$ and 4 from our model. We present the results for $c$ providing the best fit with the experiments. We notice that odd-parity states are associated to larger values for $c>c_{\text {crit }}$, while evenparity states are associated with relative smaller values for $c$.

In Figs. 2 and 3 we present medium heavy nuclei where the $\alpha$-daughter quasimolecular rotational bands are manifest. From the slope of the mean positions of the rotational bands we obtain the value of the moment of inertia: $\mathcal{I}=3.3 \pm$ $0.2 \hbar^{2} / \mathrm{MeV}$ for ${ }^{28} \mathrm{Si}$ and $\mathcal{I}=3.8 \pm 0.3 \hbar^{2} / \mathrm{MeV}$ for ${ }^{36} \mathrm{Ar}$, which are almost half of the values for rigid rotor configuration for the given masses and radii.

In Fig. 2 we present a comparison between experimental energy spectra [46] of resonant states for the elastic collision of $\alpha$-particles on ${ }^{28} \mathrm{Si}$ targets and the theoretical Bethe spectra (10) for $N=3$ and 4 and with the parameter $c$ chosen to provide the best fit with experiments. We remark that the odd angular momentum states are associated with larger values of $c$. Also, the states with $J=6,7$ have the best theoretical fit for rapidity $N=3$, while all the other states are related to $N=4$.

In Fig. 3 we present another set of experimental energy spectra [54] for the resonant states during collisions of $\alpha$-particles on ${ }^{36} \mathrm{Ar}$ targets, in comparison with the theoretical Bethe spectra (10). One notices more theoretical energy levels than the experimental spectra, but this may be explained by the limitations in data availability. There are two observations in favor of the NLS droplet model support. On one hand, experimental states with $J=5$ present an anomaly of having 


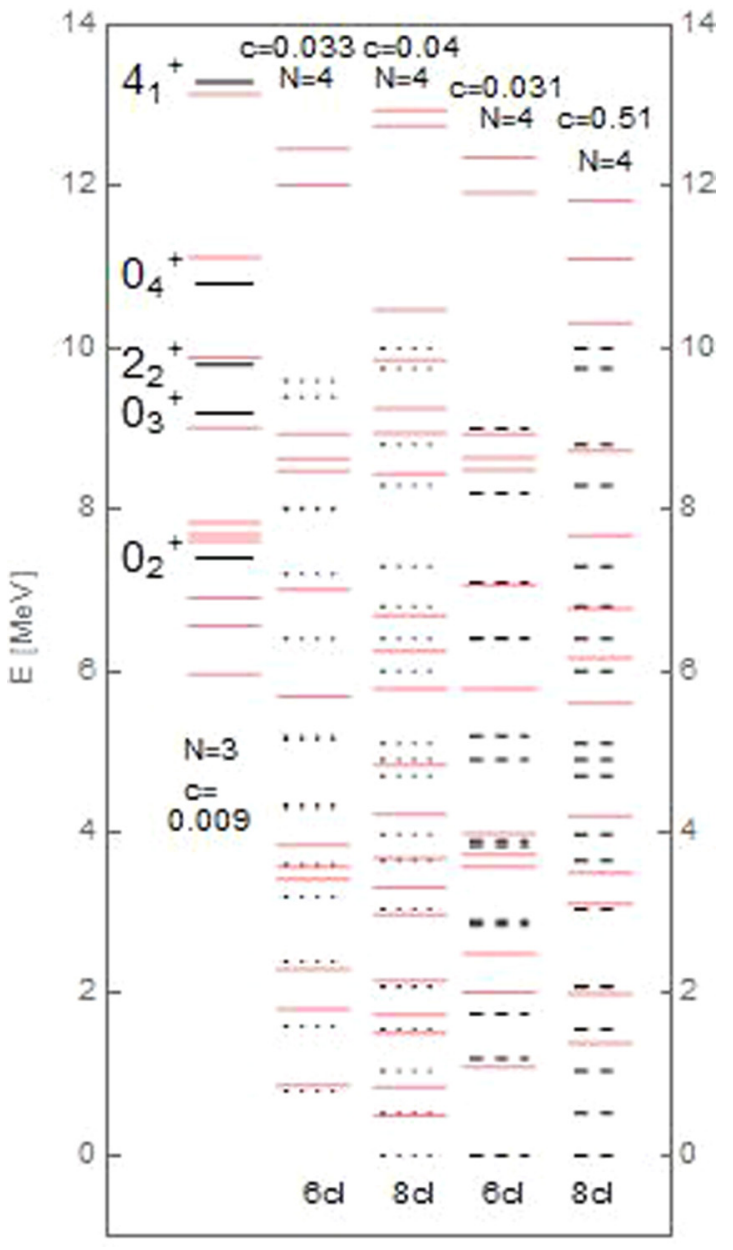

FIG. 4. Solid black: Experimental energy spectra, angular momenta, and parity of bound $\alpha$-cluster states in ${ }^{12} \mathrm{C}$. Dotted and dashed black: theoretical energy excitations calculated for $100 \%$ and $70 \%$ condensation, respectively [55]. The Hoyle state is shifted here to 0 $\mathrm{MeV}$. Under each of columns 2-5 we show the number of $\alpha$-clusters considered in the condensation model [55]: $n=6$ clusters for ${ }^{24} \mathrm{Mg}$ (columns 2,3) and $n=8$ clusters for ${ }^{32} \mathrm{~S}$ (columns 4, 5). Red: The theoretical Bethe spectra (10) for the best fit with parameters $N, c$, shown on top of each column.

very few resonances, while the NLS model also predicts a very sparse density of states when fitted at $N=3$ with energies measured at this $J$ value. On the other hand, all odd parity nuclear states from the experiment fit the best at $c \sim 1$ which represents solitons orbiting the equator of the droplet, so high values of angular momentum, while even parity states fit $c \ll 1$, which corresponds to solitons orbiting around the poles of the droplet, hence low angular momentum.

In Fig. 4 we present two types of comparisons. In the first column we plot the experimental energies of bound $\alpha$-cluster resonances in ${ }^{12} \mathrm{C}$ together with the theoretical Bethe spectra (10). We notice that, even for the best fit, our model generates the first three energy levels below the Hoyle state. In the next four columns we fit our theoretical spectra with a field theoretical super-fluid cluster model [55]. In this model, spontaneous symmetry breaks the global Wigner phase in a finite number $n$ of $\alpha$ clusters, a Bose-Einstein condensation process. We compare our Bethe spectra for $N=4$ with spectra resulting from

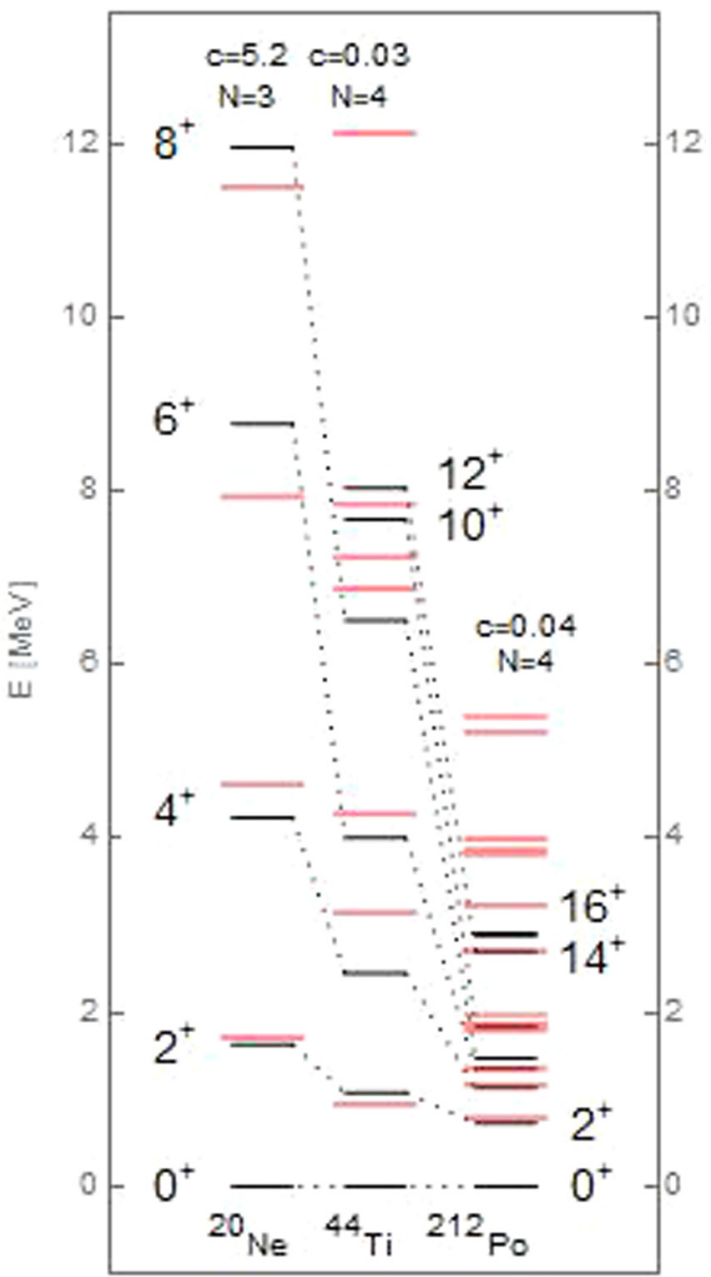

FIG. 5. Solid black: Energy, angular momentum and parity in three columns representing spectra of bound $\alpha$-cluster states, for ${ }^{20} \mathrm{Ne},{ }^{44} \mathrm{Ti}$, and ${ }^{212} \mathrm{Po}$, respectively [56]. Red: Best fit for the theoretical Bethe spectra (10) with resulting parameters $N, c$ shown on top of each column.

condensation to $n=6\left({ }^{24} \mathrm{Mg}\right)$ and $n=8\left({ }^{32} \mathrm{~S}\right) \alpha$-clusters, for two different available condensation rates of $70 \%$ and $100 \%$ calculated in [55]. In these cases, the comparison results in a good match between the two models above the Hoyle state (here $0 \mathrm{MeV}$ ).

Figure 5 represents a wide-range comparison for the energies of bound $\alpha$-cluster states: From light ${ }^{20} \mathrm{Ne}$, to medium ${ }^{44} \mathrm{Ti}$, to superheavy ${ }^{212}$ Po nuclei, with the theoretical Bethe spectra (10). While we do not have a perfect match for each spectral line, the structure and density of spectral lines is matched surprisingly well for all masses, energies, and angular momenta, in spite of the fact that we have only two free parameters $E_{0}, c$, plus the choice of which value for rapidity $N$ and which spectral tower to use. We note that lighter nuclei are fitted better by smaller rapidity $(N=3)$ and larger form parameter $(c \sim 5)$, while heavier nuclei are fitted better by larger rapidity $(N=4)$ and smaller values for the form parameter $(c \ll 1)$, while obeying the same rule: The larger the atomic mass or number of $\alpha$ clusters, the larger $c$ values fit better. Moreover, these experimental spectra were used for comparisons with the predictions of the quartet model [56] 
or the density-dependent cluster model plus the two-potential approach for heavy nuclei [60]. The intrinsic wave function of the quartet acquires a cluster configuration, when it orbits a radius above the core nucleus, similar to the surface formation of the soliton solution, Eq. (7), in the defocusing NLS nuclear shape model.

\section{CONCLUSIONS}

In this paper we have developed an asymptotic description of azimuthal envelope solitons on spherical liquid layers as solutions of defocusing nonlinear Schrödinger equation. The quantum dynamics is analyzed using the algebraic Bethe ansatz, showing a spectrum of a rigid rotor for weak nonlinearity (measured by the coefficient of nonlinear term in the NLS equation) and an oscillatory-type spectrum for strong nonlinearity. On the other hand the approximation used to get the
NLS equation needs to be improved to include the evolution in the polar coordinate as well. We expect that fully localized lump-type solutions to move on the surface of the spherical liquid. However, because of the spherical geometry it is almost sure that such an equation will be a nonautonomous one, and only numerical simulations will show interesting facts. In order to validate the model, we compare its theoretical predictions in terms of energy excitations with a large set of nuclear experimental data of energies of cluster resonant states or elastic collisions, for a variety of atomic masses from light to superheavy nuclei, and for the corresponding angular momenta and parities. The NLS models seem to offer a good fit with the structure of experimental nuclear spectra. This NLS droplet model is intended to elaborate on a number of theoretical questions, in an effort to be a useful complement to phenomenological and microscopic models, and help deepen our understanding on clustering phenomena and decays across the chart of nuclides.
[1] J. S. Russell, Report on waves, Proc. R. Soc. Edinburgh 11, 319 (1844).

[2] P. G. Kevrekidis, J. Cuevas-Maraver, and A. Saxenas, Emerging Frontiers in Nonlinear Science, Nonlinear Systems and Complexity (Springer-Verlag, New York, 2020).

[3] E. B. Kolomeisky and E. Sarabamoun, Solitons in the Einstein universe, Phys. Rev. D 101, 043515 (2020).

[4] H. Y. Schive, M. H. Liao, T. P. Woo, S. K. Wong, T. Chiueh, T. Broadhurst, and W. Y. Pauchy Hwang, Understanding the Core-Halo Relation of Quantum Wave Dark Matter from 3D Simulations, Phys. Rev. Lett. 113, 261302 (2014).

[5] P. Forgács and Á. Lukács, Nontopological solitons in Abelian gauge theories coupled to $u(1) \times u(1)$ symmetric scalar fields, Phys. Rev. D 102, 076017 (2020).

[6] D. Luo, Y. Jin, J. H. V. Nguyen, B. A. Malomed, O. V. Marchukov, V. A. Yurovsky, V. Dunjko, M. Olshanii, and R. G. Hulet, Creation and Characterization of Matter-Wave Breathers, Phys. Rev. Lett. 125, 183902 (2020).

[7] A. Farolfi, D. Trypogeorgos, C. Mordini, G. Lamporesi, and G. Ferrari, Observation of Magnetic Solitons in Two-Component Bose-Einstein Condensates, Phys. Rev. Lett. 125, 030401 (2020).

[8] A. Romero-Ros, G. C. Katsimiga, P. G. Kevrekidis, B. Prinari, G. Biondini, and P. Schmelcher, On-demand generation of dark soliton trains in Bose-Einstein condensates, Phys. Rev. A 103, 023329 (2021).

[9] O. R. Sulymenko, O. V. Prokopenko, V. S. Tyberkevych, A. N. Slavin, and A. A. Serga, Bullets and droplets: Two-dimensional spin-wave solitons in modern magnonics, Low Temp. Phys. 44, 602 (2018).

[10] B. Blasius, L. Rudolf, G. Weithoff, U. Gaedke, and G. F. Fussmann, Long-term cyclic persistence in an experimental predator-prey system, Nature (London) 577, 226 (2020).

[11] Y. Song, X. Shi, C. Wu, D. Tang, and H. Zhang, Recent progress of study on optical solitons in fiber lasers, Appl. Phys. Rev. 6, 021313 (2019).

[12] J. M. Dudley, G. Genty, A. Mussot, A. Chabchoub, and F. Dias, Rogue waves and analogies in optics and oceanography, Nat. Rev. Phys. 1, 675 (2019).
[13] G. Marcucci, D. Pierangeli, A. J. Agranat, R.-K. Lee, E. DelRe, and C. Conti, Topological control of extreme waves, Nat. Comm. 10, 5090 (2019).

[14] Z. Y. Sun and X. Yu, Anomalous diffusion of discrete solitons driven by evolving disorder, Phys. Rev. E 101, 062211 (2020).

[15] P. Suret, A. Tikan, F. Bonnefoy, F. Copie, G. Ducrozet, A. Gelash, G. Prabhudesai, G. Michel, A. Cazaubiel, E. Falcon, G. El, and S. Randoux, Nonlinear Spectral Synthesis of Soliton Gas in Deep-Water Surface Gravity Waves, Phys. Rev. Lett. 125, 264101 (2020).

[16] S. Chouli and P. Grelu, Rains of solitons in a fiber laser, Optic Express 17, 11776 (2009).

[17] N. A. Silva, T. D. Ferreira, and A. Guerreiro, Reservoir computing with solitons, New J. Phys. 23, 023013 (2021).

[18] G. Marcucci, D. Pierangeli, and C. Conti, Theory of Neuromorphic Computing by Waves: Machine Learning by Rogue Waves, Dispersive Shocks, and Solitons, Phys. Rev. Lett. 125, 093901 (2020).

[19] I. Komis, S. Sardelis, Z. H. Musslimani, and K. G. Makris, Equal-intensity waves in non-Hermitian media, Phys. Rev. E 102, 032203 (2020).

[20] C. Markos, J. C. Travers, A. Abdolvand, B. J. Eggleton, and O. Bang, Hybrid photonic-crystal fiber, Rev. Mod. Phys. 89, 045003 (2017).

[21] A. Ludu and J. P. Draayer, Nonlinear Modes of Liquid Drops as Solitary Waves, Phys. Rev. Lett. 80, 2125 (1998).

[22] A. Ludu, Nonlinear Waves and Solitons on Contours and Closed Surfaces, 2nd ed., Springer Series in Synergetics (SpringerVerlag, New York, 2012).

[23] W. Weng, R. Bouchard, E. Lucas, E. Obrzud, T. Herr, and T. J. Kippenberg, Heteronuclear soliton molecules in optical microresonators, Nat. Comm. 11, 2402 (2020).

[24] V. Voropaev, A. Donodin, A. Voronets, D. Vlasov, V. Lazarev, M. Tarabrin, and A. Krylov, Generation of multi-solitons and noise-like pulses in a high-powered thulium-doped all-fiber ring oscillator, Sci. Rep. 9, 18369 (2019).

[25] A. S. Desyatnikov and Y. S. Kivshar, Rotating Optical Soliton Clusters, Phys. Rev. Lett 88, 053901 (2002), Y. He, D. Mihalache, B. A. Malomed, Y. Qiu, Z. Chen, and Y. Li, 
Generation of polygonal soliton clusters and fundamental solitons in dissipative systems by necklace-ring beams with radial-azimuthal phase modulation, Phys. Rev. E 85, 066206 (2012).

[26] C. Wexler and A. T. Dorsey, Contour dynamics, waves, and solitons in the quantum Hall effect, Phys. Rev. B 60, 10971 (1999).

[27] Y. V. Kartashov and D. A. Zezyulin, Stable Multi-Ring and Rotating Solitons in Two-Dimensional Spin-Orbit Coupled Bose-Einstein Condensates with a Radially-Periodic Potential, Phys. Rev. Lett. 122, 123201 (2019).

[28] E. J. Yarmchuk, M. J. V. Gordon, and R. E. Packard, Observation of Stationary Vortex Array in Rotating Superfluid Helium, Phys. Rev. Lett. 43, 214 (1979).

[29] A. Ludu and A. Raghavendra, Rotating hollow patterns in fluids, Appl. Num. Math. 141, 167 (2019).

[30] H. Ait Abderrahmane, P. S. Sedeh, H. D. Ng, and G. H. Vatistas, Rotating polygonal depression soliton clusters on the inner surface of a liquid ring, Phys. Rev. E 99, 023110 (2019).

[31] F. M. Russell, Y. Zolotaryuk, J. C. Eilbeck, and T. Dauxois, Moving breathers in a chain of magnetic pendulums, Phys. Rev. B 55, 6304 (1997).

[32] M. Amaouche, H. A. Abderrahmane, and G. H.Vatistas, Nonlinear modes in the hollow-cores of liquid vortices, Euro. J. Mech. 41, 133 (2013).

[33] R. J. A. Hill and L. Eaves, Nonaxisymmetric Shapes of a Magnetically Levitated and Spinning Water Droplet, Phys. Rev. Lett. 101, 234501 (2008).

[34] S. Perrard, Y. Couder, E. Fort, and L. Limat, Leidenfrost levitated liquid tori, Europhys. Lett. 100, 54006 (2012).

[35] A. Duchesne, T. Bohr, B. Bohr, and L. Tophoj, Nitrogen swirl: Creating rotating polygons in a boiling liquid, Phys. Rev. Fluids 4, 100507 (2019).

[36] R. Bergmann, L. Tophæoj, T. A. M. Homan, P. Hersen, A. Andersen, and T. Bohr, Polygon formation and surface flow on a rotating fluid surface, J. Fluid Mech. 679, 415 (2011).

[37] D. Fabre and J. Mougel, Generation of three-dimensional patterns through wave interaction in a model of free surface swirling flow, Fluid Dyn. Res. 46, 061415 (2014).

[38] T. R. N. Jansson, M. P. Haspang, K. H. Jensen, P. Hersen, and T. Bohr, Polygons on a Rotating Fluid Surface, Phys. Rev. Lett. 96, 174502 (2006).

[39] Y. A. Stepanyants, Dynamics of internal envelope solitons in a rotating fluid of a variable depth, Fluids 4, 56 (2019).

[40] L. Friedland and A. G. Shagalov, Resonant Formation and Control of 2D Symmetric Vortex Waves, Phys. Rev. Lett. 85, 2941 (2000).

[41] E. A. Hendricls, W. H. Schubert, Y. Chen, H. Kuo, and M. S. Peng, Hurricane eyewall evolution in a forced shallow-water model, J. Atmosph. Sci. 71, 1623 (2014).

[42] M. Allison, D. A. Godfrey, and R. F. Beebe, A wave dynamical interpretation of Saturn's polar hexagon, Science 247, 1061 (1990).

[43] L. Lindblom, J. E. Tohline, and M. Vallisneri, Nonlinear Evolution of the $r$-Modes in Neutron Stars, Phys. Rev. Lett. 86, 1152 (2001).
[44] A. Ludu, A. Săndulescu, and W. Greiner, A new large amplitude collective motion in nuclei, Int. J. Mod. Phys. E 1, 169 (1992).

[45] M. Grigorescu and A. Săndulescu, Coherent states description of $\alpha$ decay, Phys. Rev. C 48, 940 (1993).

[46] A. Ludu, A. Săndulescu, W. Greiner, K. M. Källman, M. Brenner, T. Lönnroth, and P. Manngard, $\alpha+{ }^{28}$ Si cluster structure as solitons on the nuclear surface, J. Phys. G: Nucl. Part. 21, L41 (1995)

[47] A. Ludu, A. Săndulescu, and W. Greiner, Quasimolecular resonances in the $\alpha+{ }^{20} \mathrm{Ne}$ system, J. Phys. G: Nucl. Part. 21, 1715 (1995).

[48] A. Ludu, A. Săndulescu, and W. Greiner, Nonlinear liquid drop model. Cnoidal waves, J. Phys. G: Nucl. Part. 23, 343 (1997).

[49] R. Gherghescu, A. Ludu, and J. P. Draayer, Soliton excitations as emitted clusters on nuclear surfaces, J. Phys. G: Nucl. Part. 27, 63 (2001).

[50] Z. Yuan, J. Wang, M. Chu, G. Xia, and Z. Zheng, Loss of stability of a solitary wave through exciting a cnoidal wave on a Fermi-Pasta-Ulam ring, Phys. Rev. E 88, 042901 (2013).

[51] E. D. Belokolos, A. I. Bobenko, V. S. Enolskii, and A. R. Its, Algebro-Geometric Approach to Nonlinear Integrable Equations (Springer, Berlin, 1994).

[52] V. E. Korepin, N. M. Bogoliubov, and A. G. Izergin, Quantum Inverse Scattering Method and Correlation Functions (Cambridge University Press, Cambridge, 2020).

[53] J. Sato, R. Kanamoto, E. Kaminishi, and T. Deguchi, Exact Relaxation Dynamics of a Localized Many-Body State in the 1D Bose Gas, Phys. Rev. Lett. 108, 110401 (2012).

[54] M. Norrby et al.,Elastic alpha-particle resonances as evidence of clustering at high excitation in ${ }^{40} \mathrm{Ca}$, Eur. Phys. J. 47, 96 (2011).

[55] R. Katsuragi, Y. Kazama, J. Takahashi, Y. Nakamura, Y. Yamanaka, and S. Ohkubo, Bose-Einstein condensation of $\alpha$ clusters and new soft mode in ${ }^{12} \mathrm{C}_{-}{ }^{52} \mathrm{Fe} 4 \mathrm{~N}$ nuclei in a fieldtheoretical superfluid cluster model, Phys. Rev. C 98, 044303 (2018).

[56] D. Bai, Z. Ren, and G. Röpke, Alpha clustering from the quartet model, Phys. Rev. C 99, 034305 (2019).

[57] U. Abbondanno, N. Cindro, and P. M. Milazzo, Quasimolecular interpretation of $\alpha+$ nucleus resonances, Nuovo Cimento A 110, 955 (1997).

[58] C. Beck et al., Clusters in light nuclei, Acta Phys. Polon. B 42 747 (2011).

[59] D. S. Delion, A. Dumitrescu, and V. V. Baran, Theoretical investigation of $\alpha$-like quasimolecules in heavy nuclei, Phys. Rev. C 97, 064303 (2018).

[60] D. Bai and Z. Ren, $\alpha$ clustering slightly above ${ }^{100} \mathrm{Sn}$ in the light of the new experimental data on the superallowed $\alpha$ decay, Eur. Phys. J. A 54, 220 (2018).

[61] V. G. Kartavenko, K. A. Gridnev, and W. Greiner, Nonlinear evolution of the axisymmetric nuclear surface, Phys. Atomic Nuclei 65, 637 (2002) 